\title{
Structure-based virtual screening and molecular dynamics simulations for detecting novel candidates as FGFR1 inhibitors
}

\author{
Güneş ÇOBAN 1 * iD \\ 1 Department of Pharmaceutical Chemistry, Faculty of Pharmacy, Ege University, Bornova 35040 İzmir, Turkey. \\ * Corresponding Author. E-mail: gunes.coban@ege.edu.tr (G.Ç.); Tel. +090-232-311 3277.
}

Received: 02 October 2020 / Revised: 19 December 2020 / Accepted: 19 March 2021

\begin{abstract}
Structure-based virtual screening (SBVS) was performed to predict lead compounds for fibroblast growth factor receptor 1 (FGFR1) inhibition screening the kinase inhibitor database taken from ChEMBL. The prepared kinase inhibitor database consisted of 48017 ligands were screened in ATP binding site of FGFR1 by CCDC Gold software using virtual screening parameters to filter out. After then, 720 ligands were docked inside FGFR1 using default docking parameters of CCDC Gold software. The GOLD fitness score values of 70 and 80 was used a threshold value for screening and docking process, respectively. The ligands as reduced numbers to twenty-two in terms of docking results were utilized to calculate MMGBSA free binding energy from $10 \mathrm{~ns}$ molecular dynamics simulations (MDS). For refinement of results, six of twenty-two ligands which have better calculated MMGBSA free binding energy were exposed to $100 \mathrm{~ns}$ MDS. Then, $100 \mathrm{~ns}$ MDS trajectories of six compounds were used to calculate MMGBSA free binding energy, and MDS were expended to $250 \mathrm{~ns}$ for three ligands which have highest free binding energies. By free binding energies calculated from expanded MDS, were used to predict the most promising candidates (compounds G9 and G10) for FGFR1 inhibition. Structure stability, binding modes and energy decomposition analysis were performed to insight into dynamic behaviors of compounds G9 and G10 inside FGFR-1.
\end{abstract}

KEYWORDS: FGFR1; virtual screening; molecular dynamics simulations; free binding energy.

\section{INTRODUCTION}

The fibroblast growth factor receptors (FGFRs) consist of four members named FGFR1-4 and are a subclass of the tyrosine kinases (RTKs) family. Although FGFRs expression revealed in embryogenesis causes cell differentiation, migration, morphogenesis and proliferation, in the adult organism provides angiogenesis and organogenesis, and development of the skeleton. [1]. The FGFRs display a sequence similarity with 75$92 \%$ at the tyrosine kinase domain in humans. This similarity has seemed between FGFR1 and FGFR2 more than other isoforms [1,2]. FGFR monomers are comprised of an intracellular tyrosine kinase domain, an extracellular ligand-binding domain, and a single-pass transmembrane domain, that situates between these domains. The receptor dimerization of FGFRs activated by ligand binding, initiates the downstream Ras-RafMapK, PI3K-Akt, STATs, and PLC Y signaling cascade regulating the crucial physiological processes [3-5]. The abnormal expression of FGFRs in cells was reported to related to various cancer types as gastric, bladder, breast, cervical, lung, liver, urothelial, endometrial, and multiple myeloma [4, 6-11]. It has been specified that the amplification and mutation of FGFR1 gene are manifested in various diseases as breast cancer, ovary cancer, gastric cancer, lung adenocarcinoma, oral squamous cell and urothelial carcinomas and, additionally, FGFR1 mutation is associated with Pfeiffer, Crouzon, Jackson-Weiss, Apert and Kallmann syndromes [6, 8-10, 12-16].

FGFR1 has a preserved ATP binding site in large, that consist of five regions named adenine region, hydrophobic regions I and II, ribose region and phosphate-binding region [5]. The adenine region (hinge region) is an important target for heterocyclic templates that imitate the adenine binding in ATP. The key residues of hinge region as Glu562 and Ala564 are crucial for the hits which target to this region. Hydrophobic region I residues such as Ala640, Val559, and Val561 (gatekeeper residue) and hydrophobic region II residue as Gly567, are crucial targets for hydrophobic groups of ATP competitive inhibitors. Besides, hydrophobic region I residues as Asp641, Glu531, Lys514, are the targets to form hydrogen bonding with several FGFR

How to cite this article: Çoban G. Structure-based virtual screening and molecular dynamics simulations for detecting novel candidates as FGFR1 inhibitors. J Res Pharm. 2021; 25(3): 318-330. 
inhibitors. The nucleotide domain, which is rarely used for binding by inhibitors, exists next to the hydrophobic region II and Asn568 is the key residue of this region for ligand binding. Lastly, the phosphate region is the settling location of phosphates in the ATP (Figure 1) [5, 17].

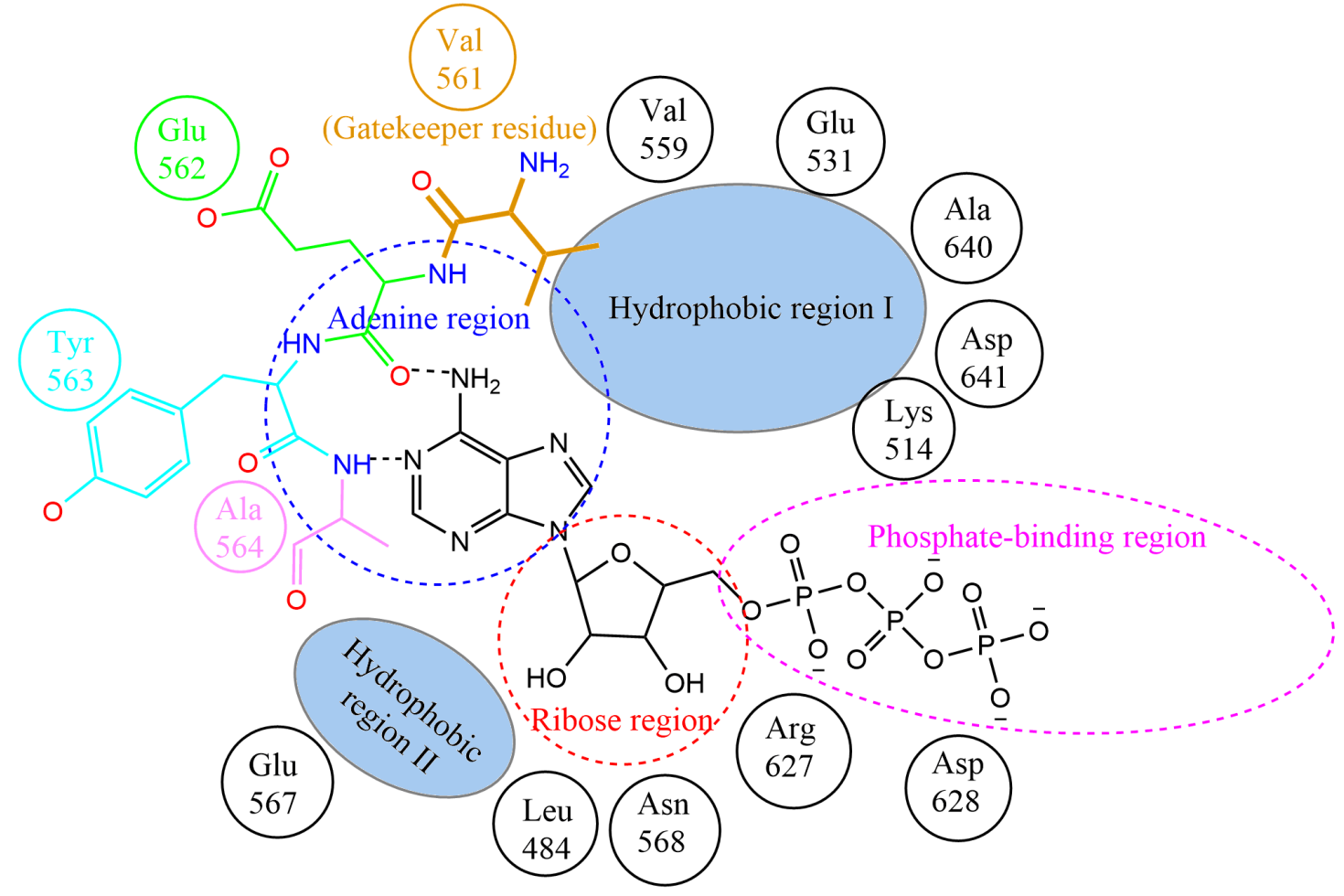

Figure 1. ATP binding site region of FGFR1 and ATP interactions with the hinge residues of FGFR1. Hydrogen bonds are represented by black dashed lines.

Crystal structure of FGFR1 kinase domain was reported for the first time by Mohammadi and coworkers in 1996 [18]. In the following study of this group, they had reported the crystal structures of tyrosine kinase domain in complex with SU4984 (1) and SU5402 (2) (Figure 2, Figure S1) [19]. In the title study, SU4984 (1) and SU5402 (2) had interacted with adenin region residues as Glu562 and Ala564. Besides, United States Food and Drug Administration (FDA) approved FGFR1 inhibitors as erdafitinib (3) and ponatinib (4) had formed hydrogen bonds with Ala564 and hydrophobic region residue as Asp641 in the crystal structure of ligandsrelated proteins (Figure 2, Figure S1) [20, 21]. Other FDA approved FGFR1 inhibitor as lenvatinib (5) had formed hydrogen bonds with Ala564 and hydrophobic region residue as Glu531 in crystal structure of lenvatinib-FGFR1 (Figure 2, Figure S1) [22]. Dovitinib (6), phase III stage compound, was reported to form hydrogen bonds with Ala564 and Glu562 (Figure 2, Figure S1) [23]. These examples display that hinge region residues as Ala564 and Glu562, and hydrophobic region I residues as Glu531 and Asp641 are important targets for the inhibition of FGFR1.

Virtual screening is a popular and effective computational process for a quick appraisal of large molecule databases to help the detection of biologically potent compounds. Structure-based virtual screening (SBVS) is a virtual screening technique that is able to identify the binding orientations of hit molecules in the active domain of a target protein using molecular docking tools. With the help of this technique, ligand binding strength can be predicted by evaluating the formed docking poses with scoring functions. Although SBVS methods are useful tools with regards to the accuracy and efficiency for pose prediction and ligand scoring with present algorithms, and many hurdles and limitations still subsist. These limitations are the constitution of false-positive and false-negative hits in the ranked lists because of the virtual screenings like standard docking methods. In addition, the other limitation to be handled is the trustworthy simulations, which play a key role in determining molecular interaction establishment and binding strength, occurring with the ligands and receptors flexible. For this purpose, molecular dynamics, which has been extensively utilized for exploring macromolecular structures and ligand binding operations, has combined with molecular docking. This combination is enabled to enhance conformational sampling in virtual screening. Besides, molecular mechanics generalized Born surface area (MM-GBSA) and molecular mechanics Poisson Boltzmann surface area (MM-PBSA) methods, which are calculated from the trajectory of molecular dynamics simulations (MDS), 
enhance the reliability of the predictions of new bioactive molecules. Thus, this automated virtual screening workflow is a milestone to overcome the limitations of SBVS methods [24, 25].

Herein, the studied kinase inhibitor database was built from ChEMBL KinaseSARfari database (ftp://ftp.ebi.ac.uk/pub/databases/chembl/KinaseSARfari/releases/5.01). The compounds, which have a molecular weight under 250 and over 650 and / or bearing the groups are able to form covalent bonding, were removed from the prepared database. The reason of a threshold value of molecular weight was selected between 250 and 650 is the FDA approved kinase inhibitors have molecular weight with a range of 306 to 615 [26]. The molecules of the prepared database were exposed to the docking study using high throughput virtual screening (HTVS) and general docking algorithms. The top ranked docking poses of the selected compounds generated after docking study, were subjected to 10 nanoseconds (ns). Then, MM-GBSA calculations were carried out to detect the estimated binding energies of these compounds. The compounds having highest estimated binding energies calculated from $10 \mathrm{~ns}$ MD simulations were exposed to 100 nanosecond (ns) MDS. After the free energy calculations from trajectories derived from $100 \mathrm{~ns}$ MDS, three potent compounds were exposed to 250 nanosecond (ns) long MDS. Finally, binding mode of the most potent compounds, determined using free energy calculations, was predicted from $250 \mathrm{~ns}$ MDS using binding mode and energy decomposition analysis, in theoretical.

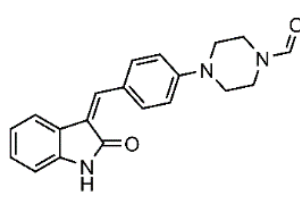

SU4984 (1)

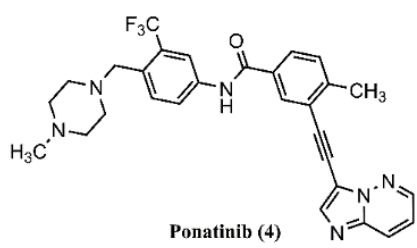

Ponatinib (4)

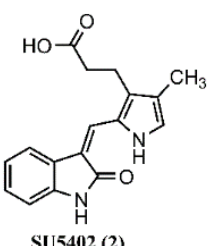

SU5402 (2)

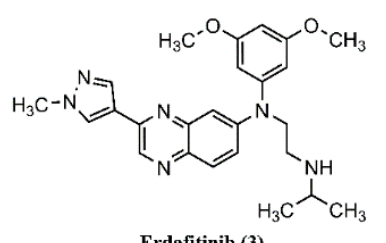

Erdafitinib (3)

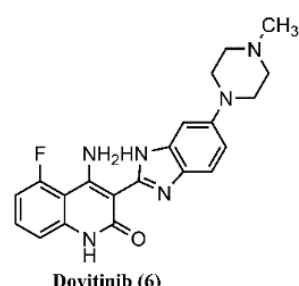

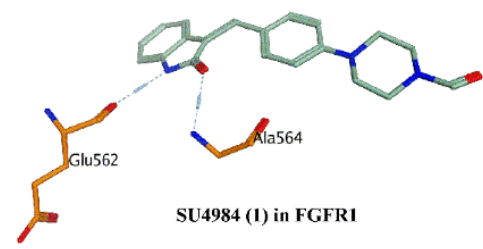
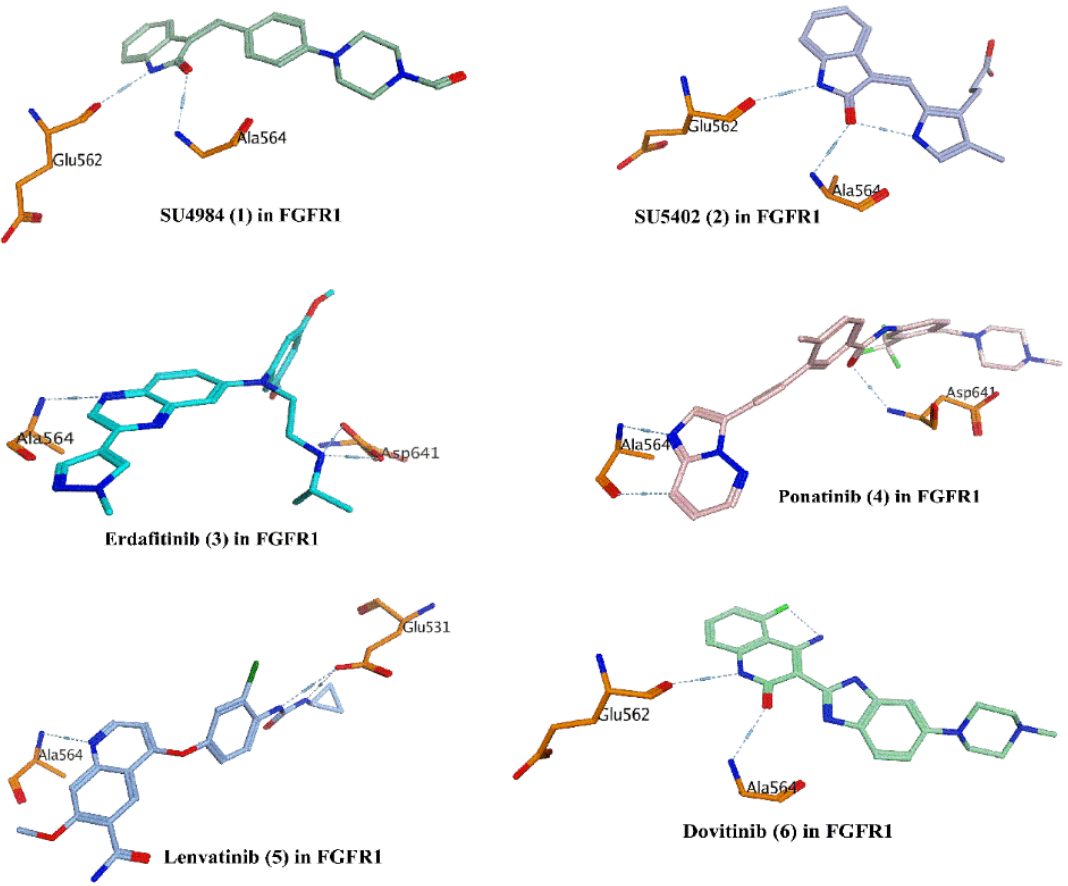

Figure 2. 2D chemical structures of some FGFR inhibitors and 3D structures of them in FGFR1. Hydrogen bonds are represented by grey dashed lines. 


\section{RESULTS AND DISCUSSION}

\subsection{Structure-based virtual screening}

In this study, 48017 ligands of prepared database were taken from the kinase inhibitor database of ChEMBL and virtually screened at the ATP binding site of FGFR1 in an attempt to predict drug candidates for FGFR1 inhibition. Screening approach was utilized to detect potential binders by comparing the calculated docking scores. Virtual screening scheme is given in Figure 3. Before the virtual screening and docking study, a validation step was conducted for the docking methods and the validation protocol was given in Supplementary material. In the first step, CCDC Gold/ virtual screening protocol was carried out to filter out low scoring ligands [27]. Then, a threshold value of GoldScore fitness as 70 was applied, the compounds which have GoldScore fitness value lower than 70, were eliminated. Separated 720 ligands were screened with CCDC Gold/default docking protocol [27]. Subsequently, the threshold value that is 70 of GoldScore fitness being increased to 80 for filtering out, was used for the selection of the ligands which were used as initial structures for calculating free binding energies from trajectories of MDS. The first ranked docking poses of twenty-two potential inhibitors acting on the active site of FGFR1 were chosen to examine in MDS. Chemical structures, the first ranked docking scores and poses of most potent inhibitors inside FGFR1 are given in Figure S2, Table 1 and Table S1, respectively.

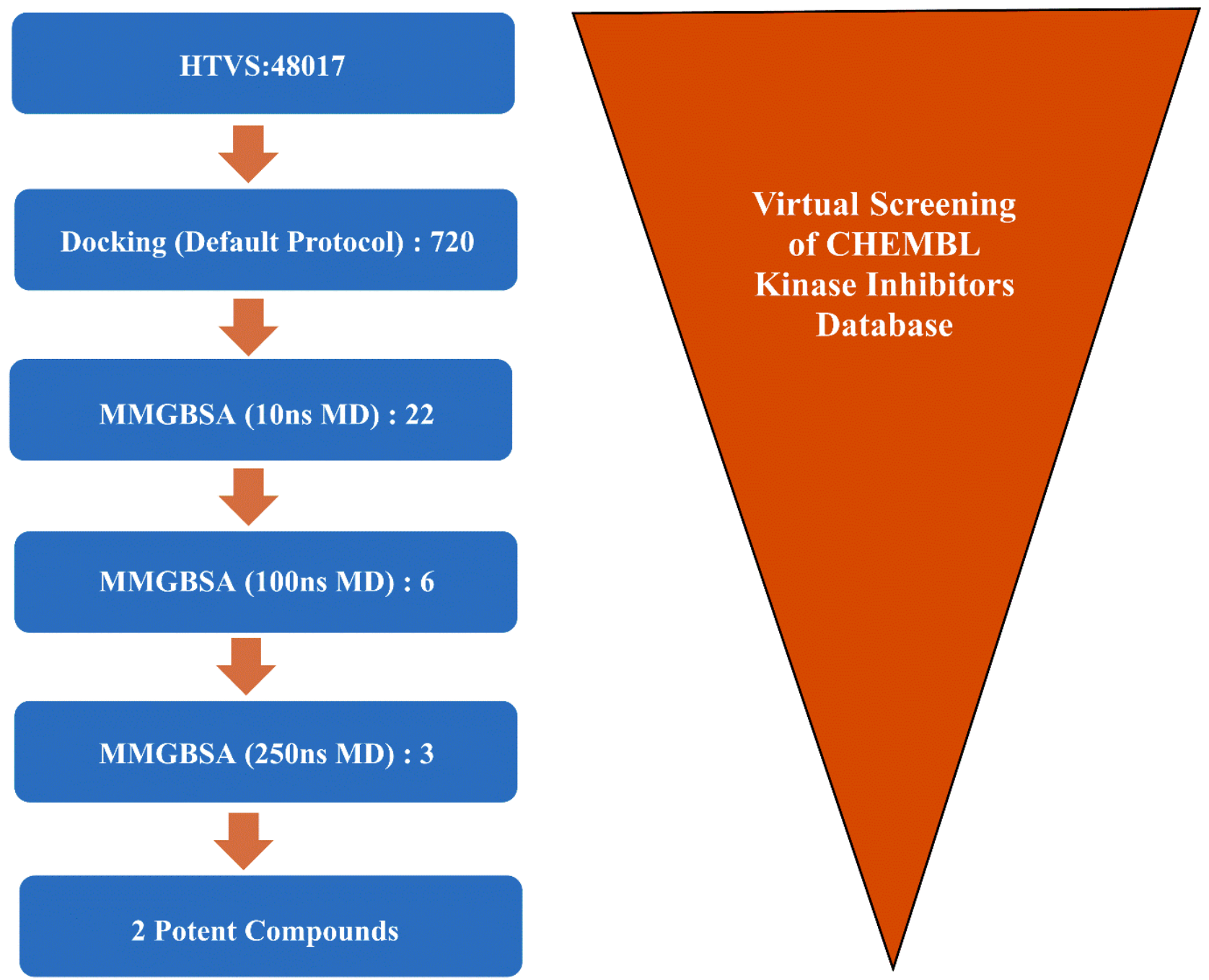

Figure 3. Numbers of used compounds in hierarchal screening approach; High-throughput virtual screening (HTVS), Docking, and MMGBSA free binding energy calculations (10ns, 100ns and 250ns, respectively). 
Table 1. The calculated docking scores (GoldScore) and the estimated MM-GBSA free binding energies of compound G1-G22 inside FGFR1 (PDB ID: 3RHX).

\begin{tabular}{ccccc}
\hline Compounds & GoldScore & $\begin{array}{c}\text { MM-GBSA for } \\
\text { 10 ns (kcal/mol) }\end{array}$ & $\begin{array}{c}\text { MM-GBSA for } \\
\text { 100 ns (kcal/mol) }\end{array}$ & $\begin{array}{c}\text { MM-GBSA for } \\
\text { 250 ns (kcal/mol) }\end{array}$ \\
\hline G1 (ID:316803) & 83.7928 & -40.0794 & & \\
G2 (ID:102217) & 83.8963 & -4.0685 & & \\
G3 (ID:1572237) & 82.3749 & -41.3008 & & \\
G4 (ID:1253838) & 83.0826 & -48.5985 & & \\
G5 (ID:1084268) & 82.9231 & -26.9538 & & \\
G6 (ID:335949) & 84.4309 & -44.2269 & & -59.5265 \\
G7 (ID:230615) & 82.2494 & -29.6935 & & \\
G8 (ID:219085) & 80.4187 & -34.4215 & & \\
G9 (ID:1088295) & 82.8773 & -53.7120 & -61.0884 & -56.2403 \\
G10 (ID:151959) & 82.0848 & -58.9669 & -68.4252 & \\
G11 (ID:218049) & 81.3193 & -47.2065 & & \\
G12 (ID:461758) & 81.9819 & -40.9900 & & \\
G13 (ID:150405) & 84.4134 & -58.3536 & -63.3880 & \\
G14 (ID:367127) & 84.1589 & -37.6440 & & \\
G15 (ID:518166) & 83.0161 & -37.9186 & & \\
G16 (ID:593813) & 85.8026 & -55.9801 & -45.6810 & \\
G17 (ID:605410) & 83.4773 & -45.2402 & & \\
G18 (ID:514509) & 83.7625 & -51.0356 & -55.4782 & \\
G19 (ID:358843) & 82.3844 & -46.2132 & & \\
G20 (ID:392998) & 81.9303 & -39.1768 & & \\
G21 (ID:359316) & 80.6725 & -51.6337 & & \\
G22 (ID:1596766) & 82.2491 & -36.4069 & & \\
\hline
\end{tabular}

\subsection{Molecular dynamics simulations and MM-GBSA free binding energy calculations}

MDS is a powerful approach that is exploited to give an insight into the dynamic behaviors of drug candidates in their targets contrary to docking studies evaluating on a single conformation in general. The free energy calculations (MM-GBSA and MM-PBSA) without entropy contribution are not true free binding energies, so these are stated as the relative free energies. For the related systems, the solute entropy is presumed to be the same for each system being compared. Therefore, the need for calculating them are removed explicitly in relative free binding calculations. These calculations using MD trajectories are applied to appraise the binding affinity of hits to their targets [28]. In this study, the first ranked conformations of title ligands inside FGFR1 formed by GOLD 5.2.1 software were used to generate MD simulations. All systems were formed by the preparing method that was specified in the experimental section. MD simulations were carried out using Amber12 software package. The protein-ligand complexes were exposed to free MDS throughout $10 \mathrm{~ns}, 100 \mathrm{~ns}$ and $250 \mathrm{~ns}$, respectively, and apo form was exposed to free MDS throughout $250 \mathrm{ns,}$ after equilibrium step.

Before the MDS generated for compounds G1-G22, MM-GBSA free binding energy calculations were executed for two FGFR inhibitors as erdafitinib and AZD4547 in FGFR1 from their 10 ns MDS trajectories and the calculation results were reported in Supplementary material. Benefiting from these calculations results, a threshold value of MM-GBSA as $-50 \mathrm{Kcal} / \mathrm{mol}$ was chosen. MDS at $10 \mathrm{~ns}$ were generated for compounds G1G22 to calculate the MM-GBSA free energies which were applied to appraise the binding affinity of hits to FGFR1. After MM-GBSA free energy calculated from 10 ns MDS, the compounds having MM-GBSA value as $-50 \mathrm{Kcal} / \mathrm{mol}$ and / or below were selected to perform for generating $100 \mathrm{~ns}$ MD simulations (Table 1). Then, the filtered three compounds according to MM-GBSA value as $-60 \mathrm{Kcal} / \mathrm{mol}$ and/or below, which calculated from 100 ns MD simulations, were exposed to 250 ns MDS. The dynamic behaviors of compound G9 reported as B-Raf kinase inhibitor and compound G10 reported as VEGFR kinase inhibitor were evaluated using free binding energy calculations, and binding mode and structural stability analysis, inside FGFR1 [29, 30]. Because of the free binding energy of compound G13 displayed a significant reduction in comparison to the free binding energy of compound G9 and G10, it has not been exposed to energy decomposition, binding mode, and structural stability analysis. 


\subsubsection{Initial structures}

Considering the estimated MM-GBSA free binding energies, compounds G9 and G10 were determined as most potent FGFR1 inhibitors among the screening compounds. Examining the docked pose (first ranking) of compound G9 inside FGFR1 generating in docking study, used in MDS study, it has seemed that compound G9 settled down into the nucleotide domain and hydrophobic region I of FGFR1. When the 3(trifluoromethyl)benzamide group of compound G9 has settled between Leu484, Tyr563, Gly567 and Asn568, the rest of the compound has occupied into the cleft residues as Phe489 and Val492, and hydrophobic region I. Regarding the docked pose (first ranking) of compound G10 inside FGFR1, it was detected that compound G10 ensconced into the hinge region and hydrophobic region I of FGFR1, and alkyl chain bearing alcohol elongated to phosphate binding region (Figure 4).

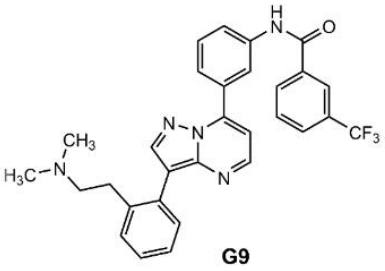

A

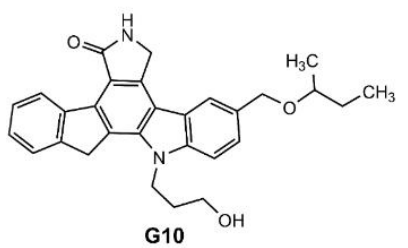

C
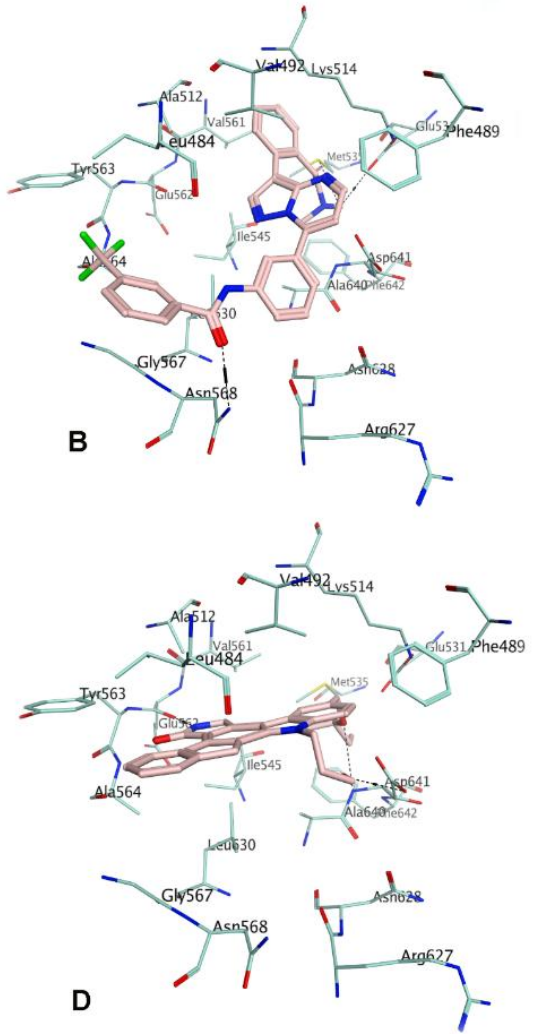

Figure 4. (A) 2D chemical structures of compound G9. (B) Docked pose of compound G9 in FGFR1. (C) 2D chemical structures of compound G10. (D) Docked pose of compound G10 in FGFR1. Light pink sticks represent compound G9 and compound G10, and light cyan sticks represent residues of FGFR1. Residues are named using three letters code. Hydrogen atoms are hidden. $\mathrm{H}$ bonds are exhibited as black dashed lines.

\subsubsection{Structural stability analysis}

The RMSD plots of ligand-FGFR1 complexes were generated from their MD trajectories to evaluate in the structural stability analysis. RMSD value of compound G9-FGFR1 complex has seemed fluctuating between around $2.0 \AA$ to $2.5 \AA$ throughout $225 \mathrm{~ns}$, and it has finished the rest of MDS with increasing the RMSD value to around $3.0 \AA$ in the RMSD plot (Figure 5). As for the RMSD value of compound G10-FGFR1 complex, it was detected gradually increasing around $1.5 \AA$ to $3.0 \AA$ from start of MDS to around $90 \mathrm{~ns}$, and fluctuating between $2.5 \AA$ to $3.0 \AA$ at between around $90 \mathrm{~ns}$ and around $160 \mathrm{~ns}$. Then by from $160 \mathrm{~ns}$, it was observed that it has kept its stability around $2.5 \AA$ through the rest of the MDS (Figure 5). In addition, it was observed that average RMSD values gradually increased around $1.0 \AA$ to $1.5 \AA$ from start of MDS to around $25 \mathrm{~ns}$, and kept around $1.5 \AA$ throughout the rest of MDS, in the RMSD plots of both ligand-FGFR1 pocket residues (Figure S3). These plots of the complexes are able to explain that compounds G9 and G10 have occupied the ATP binding site of FGFR1 and they have kept their binding orientations inside FGFR1 during the entire MDS thanks to preserving key interactions formed by active site residues. Besides, the average 
RMSD values of compounds G9 and G10 were kept their stabilities at around $0.1 \AA$ through the whole MDS (Figure 5, Figure S3).

Regarding the RMSD plot of apo form of FGFR1, it was determined that the RMSD value fluctuated to around $1.0 \AA \AA$ and $4.0 \AA \AA$ from start of MDS to around $26 \mathrm{~ns}$, and it became stable at around $2.5 \AA \hat{A}$ at the rest of MDS (Figure 5).

\subsubsection{Binding mode analysis}

Hydrogen bonding is a crucial interaction to determine ligand binding and evaluate ligand affinity to target proteins. In this study, hydrogen bonding network formed by compounds G9, and G10 with FGFR1 were determined with the analysis of MDS using Cpptraj.

According to analysis outputs, several hydrogen bonds formed by compound G9 and ATP binding site residues were detected and data about these bonds were given in Table 2. Compound G9 has formed these bonds with hydrophobic region I residues as Lys514, Glu531 and phosphate binding region residue as Asp641, nucleotide domain residue as Asn568 and hinge region residue as Ala564. The positively charged tertiary amine group of compound G9 has formed a hydrogen bond with Asp641 and preserved this bond nearly the entire MDS. Alongside hydrogen bonds formed with FGFR1 residues, hydrogen bonds were detected between compound G9 and water molecules, in addition to water-mediated bonds formed between compound G9, and Leu484, Glu562, Asn568 and Asp627 (Table 3-4, Figure 6, Figure S4). Besides of hydrogen bonding, the other crucial interactions observed between compound G9 and FGFR1 are salt bridge and the cation- $\pi$ interaction. Salt bridge has formed by the positively charged tertiary amine group and the carboxylate group of Asp641 (Figure 6, Figure S4-S6). As for the cation- $\pi$ interaction, it has formed between the protonated primary amine group of Lys514 and phenyl group of compound G9 bearing alkyl chain (Figure 6, Figure S4, S7-S8). Lastly, the other important interactions observed between compound G9 and active site residues are $\mathrm{CH}-\pi$ interactions which have been formed with alkyl side chains of Leu484, Val492, Lys514 and Asp641, and hydrophobic interactions formed with active site residues (Figure 6, Figure S4, TableS2).

With regard to evaluate binding mode of compound G10 in FGFR1, it was detected that this compound has formed strong hydrogen bonds with adenine region residues as Glu562 and Ala564, phosphate binding region residues as Arg627, Asn628 and Asp641, and nucleotide domain residue as Asn568. At the same time, it was observed that compound G10 has formed hydrogen bonds with water molecules, and formed watermediated bonds with Leu 484, Asn568 and Asp627 (Table 2-4, Figure 6, Figure S9). The other non-covalent interactions are $\mathrm{CH}-\pi$ interactions which compound G10 have been formed with alkyl side chains of Leu484, Val492, Gly567, Ala640 and phenyl group of Phe642. Besides, it was seemed that the hydrophobic interactions have formed by the active site residues of FGFR1 and compound G10 (Figure 6, Figure S9, TableS3).

G9 - FGFR1

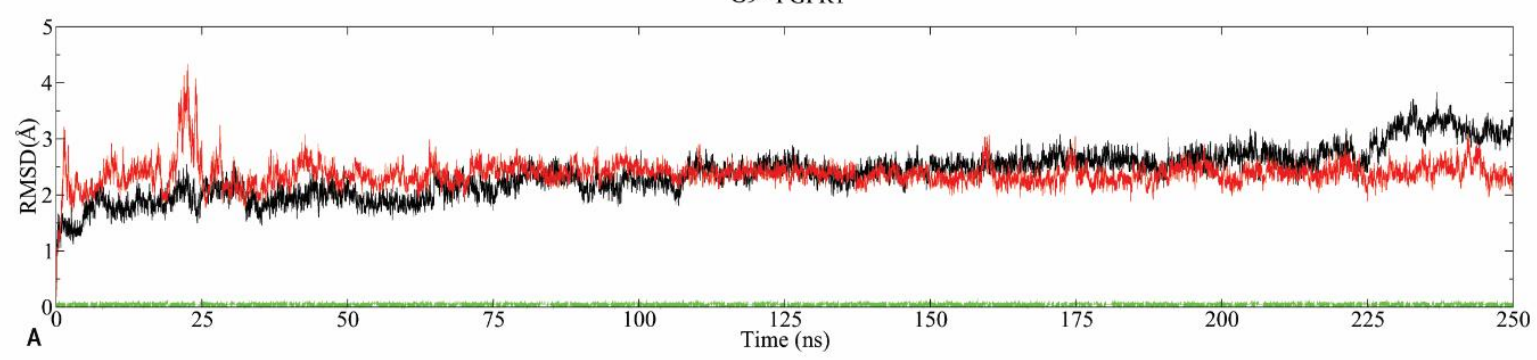

G10 - FGFR1

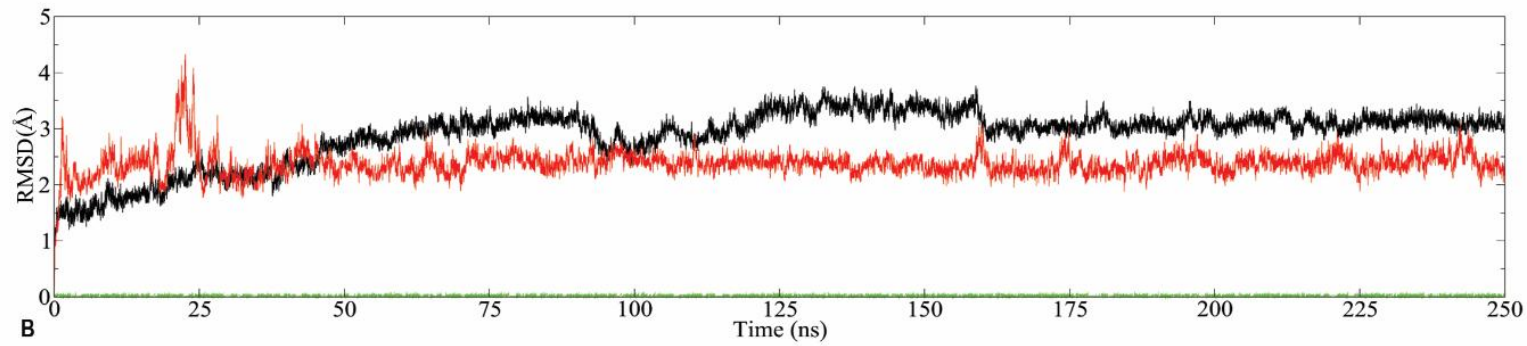

Figure 5. (A) RMSD plots of FGFR1 and compound G9-FGFR1 complex. (B) RMSD plots of FGFR1 and compound G10-FGFR1 complex. Black, red and green RMSD plots are represented for complexes, proteins and ligands, respectively. 
Table 2. Hydrogen bonding of the studied compounds in FGFR1.

\begin{tabular}{|c|c|c|c|c|c|c|c|}
\hline Compound & Acceptor & Donor-H & Donor & Count & Frac & $\begin{array}{c}\text { Average } \\
\text { Distance } \\
\text { (Angstrom) }\end{array}$ & $\begin{array}{c}\text { Average } \\
\text { Angle } \\
\text { (Degree) }\end{array}$ \\
\hline \multirow{9}{*}{ G9 } & ASP641@OD2 & $\mathrm{HN}$ & N4 & 226195 & 0.9044 & 2.7111 & 165.2919 \\
\hline & $\mathrm{O}$ & ASN568@HD22 & ASN568@ND2 & 34593 & 0.1383 & 2.8704 & 157.9902 \\
\hline & $\mathrm{N}$ & LYS514@HZ3 & LYS514@NZ & 24863 & 0.0994 & 2.9049 & 149.3728 \\
\hline & $\mathrm{N}$ & LYS514@HZ2 & LYS514@NZ & 23930 & 0.0957 & 2.9049 & 149.2816 \\
\hline & $\mathrm{N}$ & LYS514@HZ1 & LYS514@NZ & 22801 & 0.0912 & 2.9044 & 149.3372 \\
\hline & GLU531@OE1 & $\mathrm{HN}$ & $\mathrm{N} 4$ & 14247 & 0.0570 & 2.7214 & 160.2547 \\
\hline & $\mathrm{F}$ & ALA564@H & ALA564@N & 13481 & 0.0539 & 2.8906 & 152.3840 \\
\hline & $\mathrm{F} 1$ & ALA564@H & ALA564@N & 13119 & 0.0525 & 2.8900 & 151.8398 \\
\hline & F2 & ALA564@H & ALA564@N & 12809 & 0.0512 & 2.8912 & 152.0664 \\
\hline \multirow{6}{*}{ G10 } & GLU562@O & $\mathrm{H}$ & N1 & 195711 & 0.7825 & 2.8560 & 162.5503 \\
\hline & $\mathrm{O}$ & ALA564@H & ALA564@N & 180420 & 0.7214 & 2.8529 & 159.8715 \\
\hline & ARG627@O & H16 & $\mathrm{O} 2$ & 114759 & 0.4589 & 2.7332 & 160.6656 \\
\hline & O1 & ASP641@H & ASP641@N & 71928 & 0.2876 & 2.9070 & 153.3228 \\
\hline & $\mathrm{O} 2$ & ASN568@HD22 & ASN568@ND2 & 25794 & 0.1031 & 2.8832 & 152.6898 \\
\hline & ASN628@OD1 & H16 & $\mathrm{O} 2$ & 21328 & 0.0853 & 2.7508 & 159.3334 \\
\hline
\end{tabular}

Table 3. Hydrogen bonding of the studied compounds with water molecules.

\begin{tabular}{cccccccc}
\hline Compound & Acceptor & Donor-H & Donor & Count & Frac & $\begin{array}{c}\text { Average } \\
\text { Distance } \\
\text { (Angstrom) }\end{array}$ & $\begin{array}{c}\text { Average } \\
\text { Angle } \\
\text { (Degree) }\end{array}$ \\
\hline \multirow{2}{*}{ G9 } & $\mathrm{O}$ & Solvent H & Solvent Dnr & 218054 & 0.8719 & 2.7717 & 159.2080 \\
& $\mathrm{~N} 1$ & Solvent H & Solvent Dnr & 87226 & 0.3488 & 2.8813 & 160.9998 \\
& Solvent Acc & $\mathrm{H} 7$ & $\mathrm{~N} 3$ & 60551 & 0.2421 & 2.9046 & 152.5660 \\
\cline { 2 - 8 } G10 & $\mathrm{O} 2$ & Solvent H & Solvent Dnr & 106738 & 0.4268 & 2.8129 & 158.4883 \\
& Solvent Acc & $\mathrm{H} 16$ & $\mathrm{O} 2$ & 74320 & 0.2972 & 2.7710 & 161.4906 \\
\hline
\end{tabular}

Table 4. Hydrogen bonding of the studied compounds with water-mediated bridging residues of FGFR1.

\begin{tabular}{ccc}
\hline Compound & Bridging Residues & Frames \\
\hline \multirow{3}{*}{ G9 } & Arg627 & 58126 \\
& Leu484 & 18261 \\
& Glu562 & 16966 \\
\cline { 2 - 3 } G10 & Asn568 & 8688 \\
& Arg627 & 14227 \\
& Leu484 & 9908 \\
& Asn568 & 8730 \\
\hline
\end{tabular}

\subsubsection{MM-GBSA free binding energy calculations and energy decomposition analysis}

MM-GBSA free binding energy calculations for compounds G9 and G10 inside FGFR1 were yielded from $250 \mathrm{~ns}$ MDS of their complexes, and the calculation results were reported in Table 5. In the current study, MMPBSA.py.MPI module has calculated Amber MM-GBSA energy values ( $\Delta$ Gbind) of each inhibitor according to the equations given below. 


$$
\begin{gathered}
\Delta G_{\text {bind }}=\Delta E_{M M}+\Delta G_{\text {solv }} \\
\Delta E_{M M}=E_{M M C o m}-E_{M M r e c}-E_{M M} l i g \\
\Delta G_{\text {solv }}=G_{\text {solv }} \text { com }-G_{\text {solv }} r e c-G_{\text {solv }} \text { lig }
\end{gathered}
$$

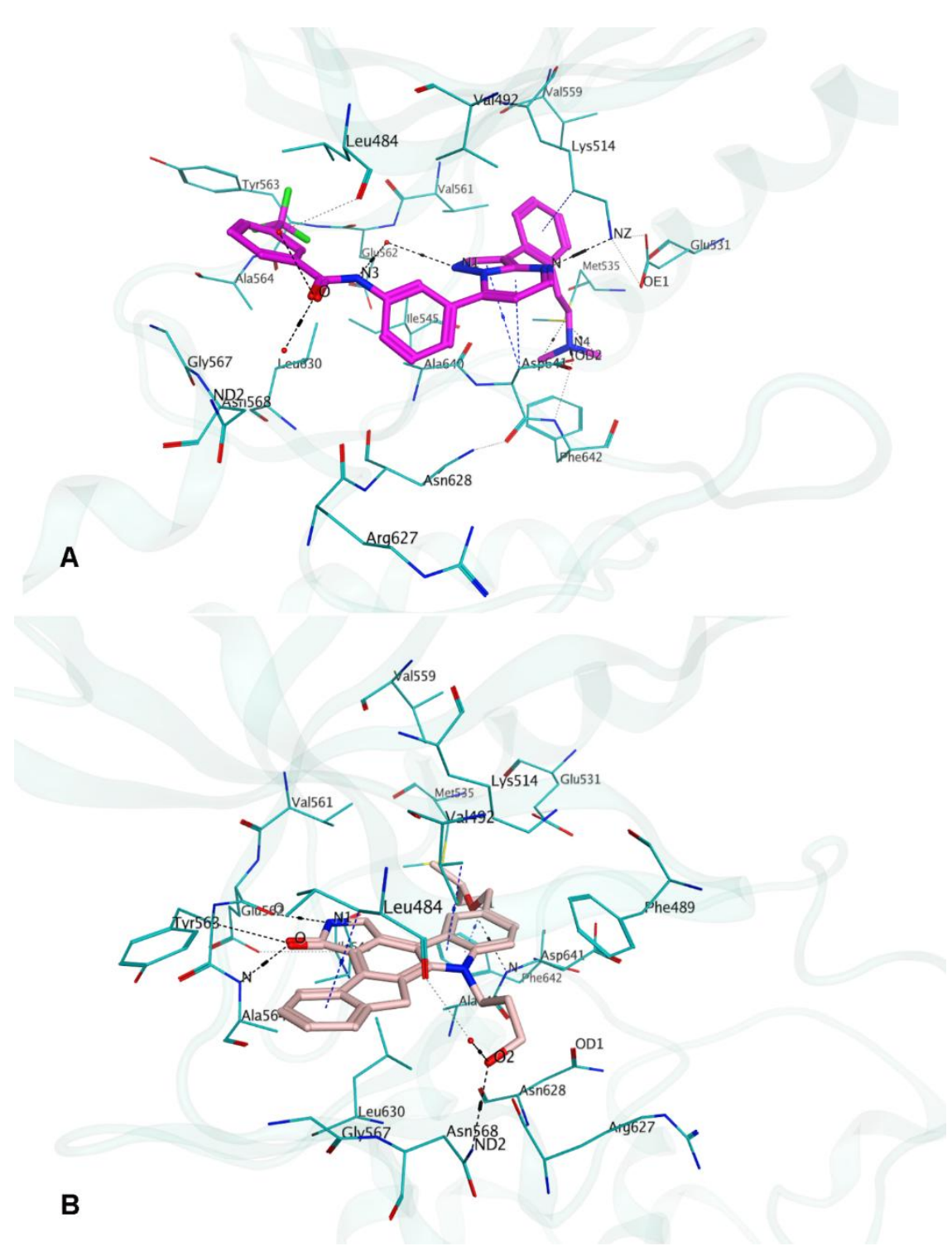

Figure 6. (A) Average structure of compound G9-FGFR1 complex generated from MDS. (B) Average structure of compound G10-FGFR1 complex generated from MDS. Magenta, pink and green sticks are represented for compound G9, compound G10 and FGFR1 residues, respectively.

In equation 1 , the molecular mechanics contribution $\left(\Delta E_{M M}\right)$ consists of the internal, electrostatic, and van der Waals contributions to binding in vacuo, and the solvation free energy contribution $\left(\Delta G_{\text {solv }}\right.$ to binding consists of polar and nonpolar solvation free energies. In equation 2 and 3, com, rec, and lig have represented to complex, receptor, and ligand, respectively.

Energy calculation results for compound G9 in FGFR1 exhibited that electrostatic energy (EEL) is the primary energy in contribution to free binding energy of title compound (Table 5). On the other hand, it was observed that van der Waals (VDWAALS) and non-polar solvation energy (ESURF) are the most preferred energy components for free binding energy, and the energy composition analysis has displayed the contribution of Leu484, Val492, Lys514, Glu531, Met535, Ile545, Val559, Val561, Tyr563, Ala564, Gly567, Asn568, Asn628, Leu630, Asp641 and Phe642 to those two energy components (Table 5, Table S2). Besides the 
energy composition analysis has supported that the total electrostatic energy expressed as the sum of electrostatic energy (EEL) and polar solvation energy (EGB) are provided by Lys514, Met535, Asn568, Asp641 and Phe642. Especially, total electrostatic energy provided by Lys514 refers to hydrogen bonding and cation$\pi$ interaction with compound G9, whereas total electrostatic energy provided by Asp641 refers to hydrogen bonding and salt bridge with compound G9. The energy composition analysis results for compound G9 were summarized in Table S2.

Table 5. MM-GBSA free binding energies (DELTA TOTAL) of the studied compounds in FGFR1.

\begin{tabular}{ccc}
\hline Compound & G9 & G10 \\
\hline VDWAALS $(\mathrm{kcal} / \mathrm{mol})$ & $-65.4163 \pm 0.0808$ & $-65.3577 \pm 0.0690$ \\
EEL $(\mathrm{kcal} / \mathrm{mol})$ & $-124.6010 \pm 0.2406$ & $-36.2831 \pm 0.1173$ \\
EGB $(\mathrm{kcal} / \mathrm{mol})$ & $138.7699 \pm 0.2256$ & $43.3652 \pm 0.0916$ \\
ESURF $(\mathrm{kcal} / \mathrm{mol})$ & $-8.2791 \pm 0.0081$ & $-7.7981 \pm 0.0059$ \\
DELTA G gas $(\mathrm{kcal} / \mathrm{mol})$ & $-190.0172 \pm 0.2539$ & $-101.6408 \pm 0.1418$ \\
DELTA G solv $(\mathrm{kcal} / \mathrm{mol})$ & $130.4908 \pm 0.2239$ & $35.5671 \pm 0.0893$ \\
DELTA TOTAL $(\mathrm{kcal} / \mathrm{mol})$ & $-59.5265 \pm 0.0951$ & $-66.0737 \pm 0.1028$ \\
\hline
\end{tabular}

Regarding the compound G10-FGFR1 complex, it was detected that van der Waals energy is the primary energy component, which contributes free binding energy for binding of compound G10 to FGFR1 (Table 5). In addition, van der Waals and non-polar solvation energy were determined that the most preferred energy components for free binding energy and the energy composition analysis has showed the contribution supplied by Leu484, Phe489, Val492, Lys514, Glu531, Met535, Ile545, Val561, Gly567, Asn568, Leu630, Ala640, Asp641 and Phe642 (Table S3). This showed that compound G10 has constituted hydrophobic interactions with, mostly, the hydrophobic region I residues. In addition to van der Waals energy, it was observed that the electrostatic energy (EEL) plays key role for the ligand binding. Total electrostatic energy (EEL and EGB) is provided by Lys514, Glu562, Tyr563, Ala564, Asn568, Arg627 and Asp641. This contribution of total electrostatic energy to free binding energy refers to hydrogen bonding formed with title residues excluded Lys514 and Tyr563. The energy composition analysis results for compound G10 were summarized in Table S3.

\section{CONCLUSION}

In summary, 48017 ligands of prepared database were taken from the kinase inhibitor database of ChEMBL. They were exposed to structure-based virtual screening and docking study to filter out. Then, selected twenty-two compounds were exposed to $10 \mathrm{~ns}$ MDS and MM-GBSA free energy calculations. After the free energy calculation results, it was considered the extension of MDS to $100 \mathrm{~ns}$ for six compounds. Following the MM-GBSA free energies calculated from $100 \mathrm{~ns}$ MD trajectories, three compounds were selected due to most promising inhibitory potencies. The selected compounds were performed to $250 \mathrm{~ns}$ MDS and MMGBSA free energy calculations. It was calculated that MM-GBSA free energy of compound G13 reduced more than the free energy of the other compounds. For these reasons, compounds G9 and G10 were selected to determine binding motif inside FGFR1 using MM-GBSA free energy calculations and structural stability, binding mode and energy decomposition analysis. Although, compound G9 and G10 have been reported as B-Raf and VEGFR kinase inhibitors by the different researching groups, respectively, there are no reported studies about FGFR1 inhibition of them. On the other hand, Gingrich and coworkers had reported FGFR1 inhibition about a derivative of compound G10. Therefore, these compounds could be considered as the proper candidates for FGFR1 inhibition.

\section{MATERIALS AND METHODS}

\subsection{Virtual screening and docking}

Partial atomic charges addition, the parametrization, and an energy minimization were implemented to the inhibitors, using MMFF94x force field with MOE.2016.08 [31, 32]. The crystal structure of FGFR1 (PDB ID: 3RHX resolved at 2.01 ^) was downloaded from the RCSB Protein Data Bank (http:/ / www.rcsb.org/pdb). Chain A of FGFR1 was preserved to use virtual screening and docking studies, and the other chain and molecules were removed. AMBER99SB force field was used to parametrize chain A [33]. Energy minimization for the protein was carried out using MOE2016.08 [32]. 
GOLD 5.2.1 software were used to dock the ligands inside FGFR1 with virtual screening and default generic algorithm parameters, respectively [27]. The inhibitors were exposed to dock in an area within a radius of $15 \AA$ centering the carboxylate carbon of Asp641 of FGFR1. GoldScore fitness function was used to generate hundred conformations per ligand. [27]. Figure 4 and figures in Table S1 and S4 were generated using MOE2016.08 program.

\subsection{Molecular dynamics simulations}

In the current study, AMBER12 was used to generate MDS of apo FGFR1 and MDS of FGFR1-ligand complexes [34]. The docked poses of compounds G1-G22 inside FGFR1 yielded from docking studies were used to prepare the starting ligand-FGFR1 systems. The antechamber with the AM1-BCC charge model was exploited to calculate the partial atomic charges of title compounds [34, 35] The xleap was exploited to the preparation of the apo FGFR1 and FGFR1-ligand complexes for energy minimizations and MDS [34]. General AMBER force field (gaff) and AMBER ff99SB force field were utilized to realize the parameterization of ligands and proteins in the complex systems [33,36]. TIP3P water molecules were used to solvate all systems in with $10 \AA$ distance between box boundary and protein surface, in an octahedral box. Sodium counter ions were employed to the neutralization of the systems as an appropriate number [37].

The energy minimizations of all systems were practiced with Sander.MPI, and then MDS of the systems were performed using pmemd.cuda [34]. An energy minimization for the initial systems were utilized in two steps in order to intercept bad steric contacts. An energy minimization with the steepest descent algorithm and conjugate gradient methods at 1000 iterations was applied to the restrained initial structures, and in second step, it was performed to the unrestrained systems with the steepest descent algorithm and conjugate gradient methods at 2500 iterations. The heating $(0.1 \mathrm{~ns})$, equilibration $(1 \mathrm{~ns})$ and production steps $(10 \mathrm{~ns})$ were practiced for the MDS of the systems. The production steps have been extended to at $100 \mathrm{~ns}$ for six compounds, and then, at $250 \mathrm{~ns}$ for three of these six compounds. In MDS, the heating step was performed with increasing the temperature of systems from 0 to $300 \mathrm{~K}$ using $10 \mathrm{kcal} / \mathrm{mol} / \AA$ restraint force allowing water molecules and ions in order to move without constraint. Then, the Langevin dynamics with a collision frequency of 1.0 $\mathrm{ps}^{-1}$ in constant volume periodic boundary were used to equilibrate the temperature of the entire systems at $300 \mathrm{~K}$. Periodic boundary conditions with constant pressure were exploited to equilibrate the pressure of systems at 1 bar using isotropic position scaling method at $300 \mathrm{~K}$. Finally, positional constraints were gradually cleared out preserving the system temperature at $300 \mathrm{~K}$ and system pressure at 1 bar. SHAKE algorithm was implemented to constrain band vibrations including hydrogen atoms in the equilibration and production steps [38].

The Particle Mesh Ewald (PME) method was applied for long-range electrostatic interactions [39]. $2 \mathrm{fs}$ was used as the time step for all MDS and nonbonded interactions were shortened using a cutoff of $10 \AA$. Visualization of trajectories was realized Xmgrace program [40]. Cpptraj was exploited to determine the hydrogen bonding using default parameters [41]. MMPBSA.py.MPI was utilized to realize free binding energy calculations and to execute energy decomposition analysis, using the Generalized-Born (GB) model from 100 spaced snapshots of free MDS. [28]. UCSF Chimera package was used to extract MD snapshots from free MDS [42]. MOE 2016.08 program was applied to create Figure 6 and figures of MD snapshots exist in the supplementary material section.

Acknowledgements: Author appreciates to Pharmaceutical Sciences Research Centre (FABAL) of Ege University Faculty of Pharmacy for molecular modelling software support.

Author contributions: Concept - G.Ç.; Design - G.Ç..; Supervision - G.Ç.; Resources - G.Ç.; Materials - G.Ç.; Data Collection and/or Processing - G.Ç.; Analysis and/or Interpretation - G.Ç.; Literature Search - G.Ç.; Writing - G.Ç.; Critical Reviews - G.Ç.

Conflict of interest statement: The author declared no conflict of interest in the manuscript.

Appendix A. Supplementary Material

Supplementary material related to this article can be accessed at https://dx.doi.org/10.29228/jrp.22 .

\section{REFERENCES}

[1] Ahmad I, Iwata T, Leung HY. Mechanisms of FGFR-mediated carcinogenesis. Biochim Biophys Acta. 2012; 1823: 850860. [CrossRef]

[2] Powers CJ, McLeskey SW, Wellstein A. Fibroblast growth factors, their receptors and signaling. Endocrine-Related Cancer. 2000; 7: 165-197. [CrossRef] 
[3] Furthauer M, Lin W, Ang SL, Thisse B, Thisse C. Sef is a feedback-induced an-tagonist of Ras/MAPK-mediated FGF signalling. Nat Cell Biol. 2002; 4: 170-174. [CrossRef]

[4] Heinzle C, Sutterlüty H, Grusch M, Grasl-Kraupp B, Berger W, Marian B. Targeting fibroblast-growth-factorreceptor-dependent signaling for cancer therapy. Expert Opin Ther Tar. 2011; 15 (7): 829-846. [CrossRef]

[5] Wang Y, Li L, Fan J, Dai Y, Jiang A, Geng M, Ai J, Duan W. Discovery of potent irreversible pan-fibroblast growth factor receptor (FGFR) inhibitors. J Med Chem. 2018; 61 (20): 9085-9104. [CrossRef]

[6] Murase H, Inokuchi M, Takagi Y, Kato K, Kojima K, Sugihara K. Prognostic significance of the co-overexpression of fibroblast growth factor receptors 1, 2 and 4 in gastric cancer. Mol Clin Oncol. 2014; 2: 509-17. [CrossRef]

[7] Nord U, Segersten J, Sandgren K, Wester C, Busch U, Menzel J, Komorowski JP, Dumanski PU, Malmström T, de Ståhl D. Focal amplifications are associated with high grade and recurrences in stage Ta bladder carcinoma. Int J Cancer. 2009; 126: 1390-1402. [CrossRef]

[8] Elbauomy Elsheikh S, Green AR, Lambros MB, Turner NC, Grainge MJ, Powe D, et al. FGFR1 amplification in breast carcinomas: a chromogenic in situ hybridisation analysis. Breast Cancer Res. 2007; 9: R23. [CrossRef]

[9] Desai A, Adjei AA. FGFR Signaling as a Target for Lung Cancer Therapy. J Thorac Oncol. 2016; 11(1): 9-20. [CrossRef]

[10] Lim S, Koh MJ, Jeong HJ, Cho NH, Choi YD, Cho DY, Lee HY, Rha SY. Fibroblast growth factor receptor 1 overexpression is associated with poor survival in patients with resected muscle invasive urothelial carcinoma. Yonsei Med J. 2016; 57(4): 831-839. [CrossRef]

[11] Li X, Guise CP, Taghipouran R, Yosaatmadja Y, Ashoorzadeh A, Paik WK, Squire CJ, Jiang S, Luo J, Xu Y, Tu ZC, Lu $X$, Ren X, Patterson AV, Smaill JB, Ding K. 2-Oxo-3,4-dihydroprimido[4, 5-d]pyrimidinyl derivatives as new irreversible pan fibroblast growth factor receptor (FGFR) inhibitors. Eur J Med Chem. 2017; 135: 531-543. [CrossRef]

[12] Gorringe KL, Jacobs S, Thompson ER, Sridhar A, Qiu W, Choong DY, Campbell IG. High-resolution single nucleotide polymorphism array analysis of epithelial ovarian cancer reveals numerous microdeletions and amplifications. Clin. Cancer Res. 2007; 13:4731-4739. [CrossRef]

[13] Kim HS, Lee SE, Bae YS, Kim DJ, Lee CG, Hur J, et al. Fibroblast growth factor receptor 1 gene amplification is associated with poor survival in patients with resected esophageal squamous cell carcinoma. Oncotarget. 2015; 6: 2562-2572. [CrossRef]

[14] Freier K, Schwaenen C, Sticht C, Flechtenmacher C, Mühling J, Hofele C, Radlwimmer B, Lichter P, Joos S. Recurrent FGFR1 amplification and high FGFR1 protein expression in oral squamous cell carcinoma (OSCC), Oral Oncol. 2007; 43: 60-66. [CrossRef]

[15] Kim SH, Ryu H, Ock C-Y, Suh KJ, Lee JY, Kim J-W, Lee J-O, Kim JW, Kim YJ, Lee K-W, Bang S-M, Kim JH, Lee JS, Ahn JB, Kim K-J, Rha SY. BGJ398, A Pan-FGFR Inhibitor, Overcomes Paclitaxel Resistance in Urothelial Carcinoma with FGFR1 Overexpression. Int J Mol Sci. 2018; 19: 3164-3179. [CrossRef]

[16] Ho HK, Yeo AHL, Kang TS, Chua BT. Current strategies for inhibiting FGFR activities in clinical applications: opportunities, challenges and toxicological considerations. Drug Discov Today. 2014; 19 (1): 51-62. [CrossRef]

[17] Liang G, Chen G, Wei X, Zhao Y, Li X. Small molecule inhibition of fibroblast growth factor receptors in cancer. Cytokine Growth F R. 2013; 24:467-475. [CrossRef]

[18] Mohammadi M, Schlessinger J, Hubbard SR. Structure of the FGF receptor tyrosine kinase domain reveals a novel autoinhibitory mechanism. Cell.1996; 86: 577-587. [CrossRef]

[19] Mohammadi M, McMahon G, Sun L, Tang C, Hirth P, Yeh BK, Hubbard SR, Schlessinger J. Structures of the tyrosine kinase domain of fibroblast growth factor receptor in complex with inhibitors. Science. 1997; 276: 955-960. [CrossRef]

[20] Patani H, Bunney TD, Thiyagarajan N, Norman RA, Ogg D, Breed J, Ashford P, Potterton A, Edwards M, Williams SV, Thomson GS, Pang CS, Knowles MA, Breeze AL, Orengo C, Phillips C, Katan M. Landscape of activating cancer mutations in FGFR kinases and their differential responses to inhibitors in clinical use. Oncotarget. 2016; 7: 2425224268. [CrossRef]

[21] Tucker JA, Klein T, Breed J, Breeze AL, Overman R, Phillips C, Norman RA. Structural insights into FGFR kinase isoform selectivity: diverse binding modes of AZD4547 and ponatinib in complex with FGFR1 and FGFR4. Structure. 2014, 22(12): 1764-1774. [CrossRef]

[22] Matsuki M, Hoshi T, Yamamoto Y, Ikemori-Kawada M, Minoshima Y, Funahashi Y, Matsui J. Lenvatinib inhibits angiogenesis and tumor fibroblast growth factor signaling pathways in human hepatocellular carcinoma models. Cancer Med. 2018; 7: 2641-2653. [CrossRef] 
[23] Klein T, Vajpai N, Phillips JJ, Davies G, Holdgate GA, Phillips C, Tucker JA, Norman RA, Scott AD, Higazi DR, Lowe D, Thompson GS, Breeze AL. Structural and dynamic insights into the energetics of activation loop rearrangement in Fgfr1 kinase. Nat Commun. 2015; 6: 7877. [CrossRef]

[24] Rastelli G. Emerging topics in structure-based virtual screening. Pharm Res. 2013; 30:1458-1463. [CrossRef]

[25] Anighoro A, Rastelli G. BEAR, a molecular docking refinement and rescoring method. Comput Mol Biosci. 2013; 3 : 27-31. [CrossRef]

[26] Roskoski Jr. R. Properties of FDA-approved small molecule protein kinase inhibitors: A 2020 update. Pharmacol Res. 2020; 152:104609. [CrossRef]

[27] Jones G, Willett P, Glen RC, Leach AR, Taylor R. Development and validation of a genetic algorithm for flexible docking. J Mol Biol. 1997; 267(3): 727-748. [CrossRef]

[28] Miller III BR, McGee Jr TD, Swails JM, Homeyer N, Gohlke H, Roitberg AE. MMPBSA.py: an efficient program for end-state free energy calculations. J Chem Theory Comput. 2012; 8: 3314-3321. [CrossRef]

[29] Berger DM, Torres N, Dutia M, Powell D, Ciszewski G, Gopalsamy A, Levin JI, Kim KH, Xu W, Wilhelm J, Hub YB, Collins K, Feldberg L, Kim S, Frommer E, Wojciechowicz D, Mallon R. Non-hinge-binding pyrazolo[1,5a]pyrimidines as potent B-Raf kinase inhibitors. Bioorg Med Chem Lett. 2009; 19: 6519-6523. [CrossRef]

[30] Gingrich DE, Reddy DR, Iqbal MA, Singh J, Aimone LD, Angeles TS, Albom M, Yang S, Ator MA, Meyer SL, Robinson C, Ruggeri BA, Dionne CA, Vaught JL, Mallamo JP, Hudkins RL. A new class of potent vascular endothelial growth factor receptor tyrosine kinase inhibitors: structure-activity relationships for a series of 9-alkoxymethyl-12(3-hydroxypropyl)indeno[2,1-a]pyrrolo[3,4-c]carbazole-5-ones and the identification of CEP-5214 and its dimethylglycine ester prodrug clinical candidate CEP-7055. J Med Chem. 2003; 46: 5375-5388. [CrossRef]

[31] Halgren TA. Merck molecular force field. I. basis, form, scope, parameterization, and performance of MMFF94*. J Comput Chem. 1996; 17: 490-519. [CrossRef]

[32] Molecular Operating Environment (MOE 2016.08) Chemical Computing Group Incorporation, 1010 Sherbrooke Street West, Suite 910, Montreal H3A 2R7, Canada.

[33] Hornak V, Abel R, Okur A, Strockbine B, Roitberg A, Simmerling C. Comparison of multiple Amber force fields and development of improved protein backbone parameters. Proteins Struct Funct Bioinf. 2006; 65: 712-725. [CrossRef]

[34] Case DA, Darden TA, Cheatham TE, Simmerling CL, Wang J, Duke RE, Luo R, Walker RC, Zhang W, Merz KM, Roberts B, Hayik S, Roitberg A, Seabra G, Swails J, Goetz AW, Kolossvảry I, Wong KF, Paesani F, Vanicek J, Wolf RM, Liu J, Wu X, Brozell SR, Steinbrecher T, Gohlke H, Cai Q, Ye X, Wang J, Hsieh MJ, Cui G, Roe DR, Mathews DH, Seetin MG, Salomon-Ferrer R, Sagui C, Babin V, Luchko T, Gusarov S, Kovalenko A, Kollman PA. 2012; Amber 12, University of California, San Francisco.

[35] Jakalian A, Bush BL, Jack DB. Fast, efficient generation of high-quality atomic charges. AM1-BCC model: I. method. J Comput Chem. 2000; 21: 132-46. [CrossRef]

[36] Wang J, Wolf RM, Caldwell JW, Kollman PA, Case DA. Development and testing of a general amber force field. J Comput Chem. 2004; 25: 1157-1174. [CrossRef]

[37] Jorgensen WL, Chandrasekhar J, Madura JD. Comparison of simple potential functions for simulating liquid water. J Chem Phys. 1983; 79: 926-935. [CrossRef]

[38] Ryckaert JP, Ciccotti G, Berendsen HJC. Numerical integration of the Cartesian equations of motion of a system with constraints: molecular dynamics of n-alkanes. J Comput Phys. 1977; 23: 327-341. [CrossRef]

[39] Essmann U, Perera L, Berkowitz ML, Darden T, Lee H, Pedersen LG. A smooth particle mesh Ewald method. J Chem Phys. 1995; 103: 8577-8593. [CrossRef]

[40] Grace Development Team, http://plasma-gate.weizmann.ac.il/Grace/(accessed on 21 May 2016).

[41] Roe DR, Cheatham III TE. PTRAJ and CPPTRAJ: software for processing and analysis of molecular dynamics trajectory data. J Chem Theory Comput. 2013; 9: 3084-3095. [CrossRef]

[42] Pettersen EF, Goddard TD, Huang CC, Couch GS, Greenblatt DM, Meng EC, Ferrin TE. UCSF Chimera -- a visualization system for exploratory research and analysis. J Comput Chem. 2004; 13: 1605-12. [CrossRef] 